\title{
FDG-Positron Emission Tomography
}

National Cancer Institute

\section{Source}

National Cancer Institute. FDG-Positron Emission Tomography. NCI Thesaurus. Code C103400.

An imaging technique that utilizes positron emission tomography to produce a threedimensional image of functional processes in the body. The system detects pairs of gamma rays emitted indirectly by a positron-emitting radionuclide (tracer), which is introduced into the body on the biologically active molecule (fludeoxyglucose) FDG. FDG is an analogue of glucose which when imaged will give tissue metabolic activity, in terms of regional glucose uptake. 